\title{
ANALYSIS AND SIMULATION OF RAYLEIGH FADING CHANNEL IN DIGITAL COMMUNICATION
}

\author{
Anila Dhingra' ${ }^{1}$ K. C. Roy $^{2}$ \\ ${ }^{I}$ Electronics \& Communication Engineering Gyan Vihar University, Jaipur, Rajasthan, India \\ anila_26@rediffmail.com \\ ${ }^{2}$ Electronics \& Communication Engineering, Kautilya Institute of Engineering \& Technology, Jaipur, Rajasthan, \\ India
}

\begin{abstract}
The objective of this paper is to analyze slow, flat, Rayleigh fading channels in modern digital communication systems having different coherent modulation techniques. Most of the packages can only be used to analyze analog communication systems, but modern systems are, increasingly, digital. Improved many-on-many (IMOM) is a two-dimensional, graphical-oriented, radio frequency modeling tool is used to determine probability of channel bit error rather than information bit error, which allows generic solutions independent of the specifics of the system under investigation as long as the particular modulation type is known. Constellation diagram of various modulation techniques, power of faded signal and signal at receiver are plotted in MATLAB environment. The Joint Tactical Information Distribution System (JTIDS) is suitable example of this analysis.
\end{abstract}

Keywords- IMOM, Rayleigh Fading, Bit Error, MATLAB

\section{INTRODUCTION}

Many wireless communications channels do not have a lineof-sight (LOS) signal path. The lack of a LOS signal path entails transmitting a signal to the receiver by a phenomenon known as multipath [1]. Multipath occurs when there are multiple paths from the transmitter to the receiver due to the reflection of the original signal off of buildings, terrain (features) or the ionosphere. The availability of a LOS does not necessarily mean that a multipath component does not exist [2]. A signal that goes through a multipath fading channel will arrive at the receiver multiple times with different amplitudes, phases and arrival times. Since multipath channels can vary significantly in terms of characteristics in an unpredictable manner, they must be modeled as a random process. The most common model for a multipath fading channel is the Rayleigh model [1, 3], where the amplitude of the received signal is modeled as a Rayleigh random variable. The probability of channel bit error equations for coherently detected binary phase-shift keying (BPSK), quadrature phase-shift keying (QPSK), Mary phase-shift keying (MPSK), M-ary quadrature amplitude modulation (MQAM) are inverted numerically [4].

JTIDS is used by the U.S. Armed Forces and its allies to support data communications needs. JTIDS serves many purposes. JTIDS is a complex system with different transmission modes, but, for this thesis only the single-pulse architecture is evaluated. The single-pulse architecture sacrifices security and jam resistance for an increased data rate. JTIDS uses Reed Solomon (RS) coding, providing secure, jam resistant, digital data communication [5]. The modulation technique used in JTIDS is cyclical code-shift keying (CCSK).

\section{ANALYSIS AND RESULT}

(A) Analysis based on Improved many-on-many A slow, flat Rayleigh fading channel is a model of a communications channel that is used when there is no lineof-sight between the transmitter and the receiver, and all of the received signal power is due to multipath. In contrast to no fading, this model represents the opposite end of the spectrum. For coherent modulation techniques, the probability of bit error for a slow, flat Rayleigh fading channel is obtained with $\alpha=0$

$p_{b}=\frac{a}{q} \int_{0}^{\infty} Q\left(\sqrt{b \gamma_{s}}\right) \frac{\sigma_{0}^{2}}{2 \sigma^{2}} \exp \left(\frac{-\gamma_{s} \sigma_{0}^{2}}{2 \sigma^{2}}\right) d \gamma_{s}$

Where

$\gamma_{b}=\frac{E_{b}}{N_{0}}=\frac{\gamma_{s}}{q}$

\section{BPSK/QPSK}

For BPSK and QPSK and a Rayleigh fading channel, (1) can be evaluated to obtain

$p_{b}=\frac{1}{2}\left(1-\sqrt{\frac{\gamma_{b}}{1+\gamma_{b}}}\right)$

thus (3) can be algebraically inverted to obtain

$\gamma_{b}=10 \log \left(\frac{\left(1-2 p_{b}\right)^{2}}{4 p_{b}-4 p_{b}^{2}}\right)$

where the result is expressed in $\mathrm{dB}$ and plotted in Figure 1. 


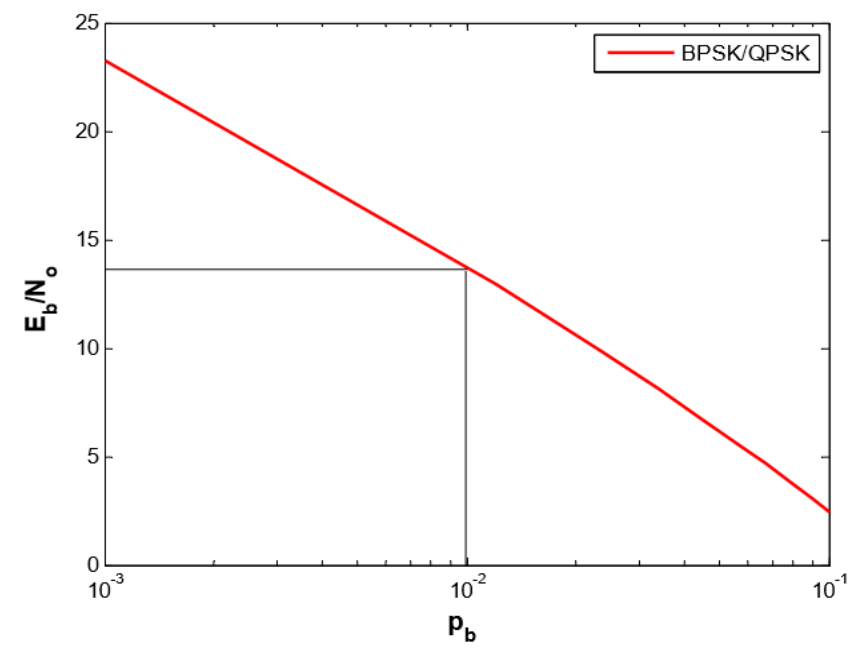

Figure 1: BPSK/QPSK performance for a Rayleigh fading channel.

The performance for BPSK/QPSK for a Rayleigh fading channel exhibits the expected performance degradation when compared to the non-fading channel performance. In order to obtain a probability of channel bit error of $p_{b}=$ $10^{-2}, 14 \mathrm{~dB}$ of signal power is required. This is significantly more than the $4.3 \mathrm{~dB}$ required for a nonfading environment.

\section{MPSK}

Evaluating (1) for MPSK and Rayleigh fading, we get [6]

$p_{b}=\frac{1}{q}\left(1-\sqrt{\frac{q \gamma_{b} \sin ^{2}(\pi / M)}{1+q \gamma_{b} \sin ^{2}(\pi / M)}}\right)$

In order to meet demands for current applications, both 8PSK and 16-PSK were evaluated with satisfactory results. After inverting (5) for 8-PSK and 16-PSK, we get $\gamma_{b}=10 \log \left(\frac{1}{8 \sin ^{2}(\pi / 8)}\right)\left(\frac{\left(1-8 p_{b}\right)^{2}}{16 p_{b}-64 p_{b}^{2}}\right)$, for 8-PSK and

$\gamma_{b}=10 \log \left(\frac{1}{16 \sin ^{2}(\pi / 16)}\right)\left(\frac{\left(1-16 p_{b}\right)^{2}}{32 p_{b}-256 p_{b}^{2}}\right)$, For 16-PS (7) for 16-PSK. (6) and (7) are plotted in Figures 2 and 3, respectively.

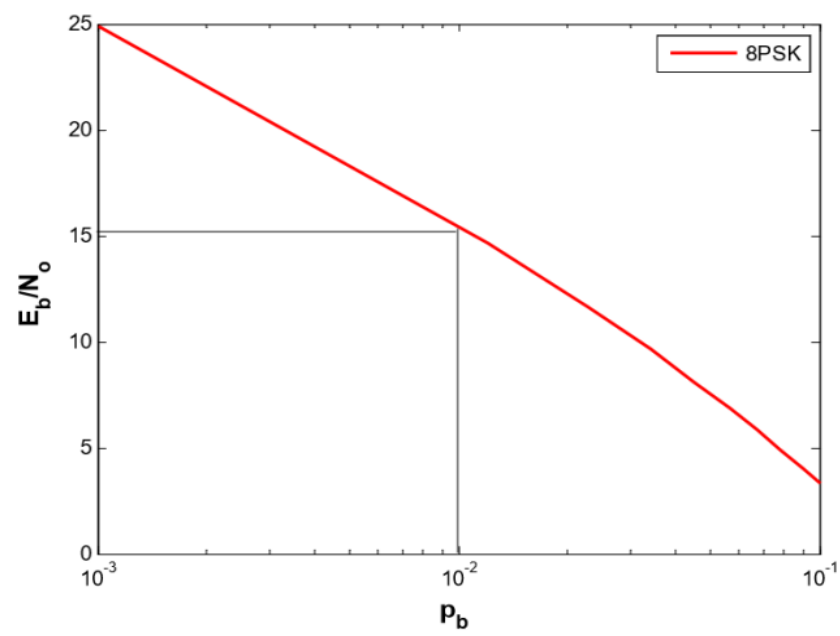

Figure 2: 8-PSK performance for a Rayleigh fading channel.

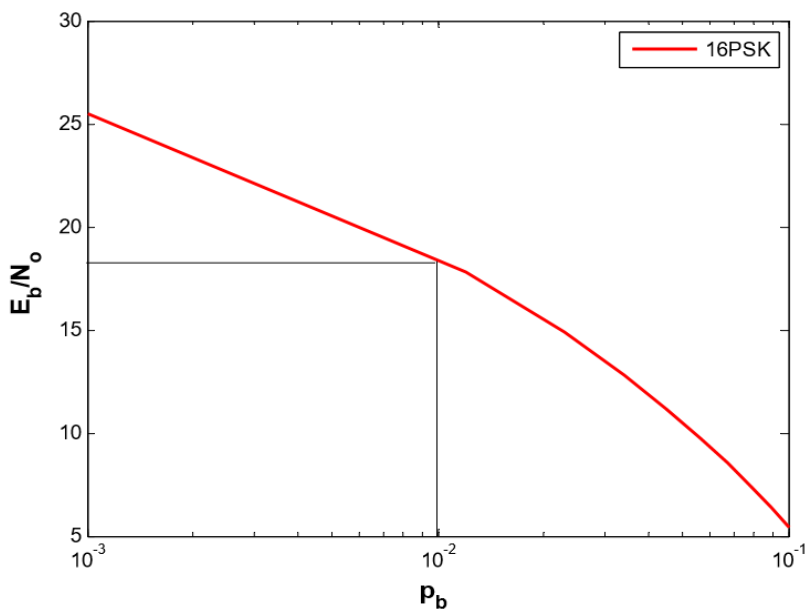

Figure 3: 16-PSK performance for a Rayleigh fading channel

For 8-PSK and 16-PSK, for a channel $p_{b}$ of $10^{-2}, E_{b} / N_{0}$ of $15.5 \mathrm{~dB}$ and $18.6 \mathrm{~dB}$, respectively, are required. As expected, the $E_{b} / N_{0}$ required increases when no line-of sight exists. This is an increase from the values of $9.7 \mathrm{~dB}$ and $15.5 \mathrm{~dB}$, respectively, for 8-PSK and 16-PSK in a non-fading channel.

\section{MQAM}

As described for a non-fading channel model, for MQAM it is necessary to examine MQAM for two variations of the modulation-type parameters, one when $\mathrm{q}$ is even and one when $\mathrm{q}$ is odd. Although both modulation-type parameters provide good results, the q-even modulation-type parameters produce slightly more accurate results [7]. Analyzing 16QAM and 64-QAM are important because they are used in the IEEE 802.11g wireless standard [3].

For MQAM with even q, (1) can be evaluated to obtain

$$
p_{b}=\frac{2}{q}\left(1-2^{\frac{-q}{2}}\right)\left(1-\sqrt{\frac{3 q \gamma_{b}}{2\left(2^{q}-1\right) 1+3 q \gamma_{b}}}\right)
$$

After inverting Equation (8), we obtain the result for 16QAM as

$$
\gamma_{b}=10 \log \left(\frac{5}{2}\right)\left(\frac{\left(1-8 / 7 p_{b}\right)^{2}}{16 / 7 p_{b}-64 / 49 p_{b}^{2}}\right)
$$

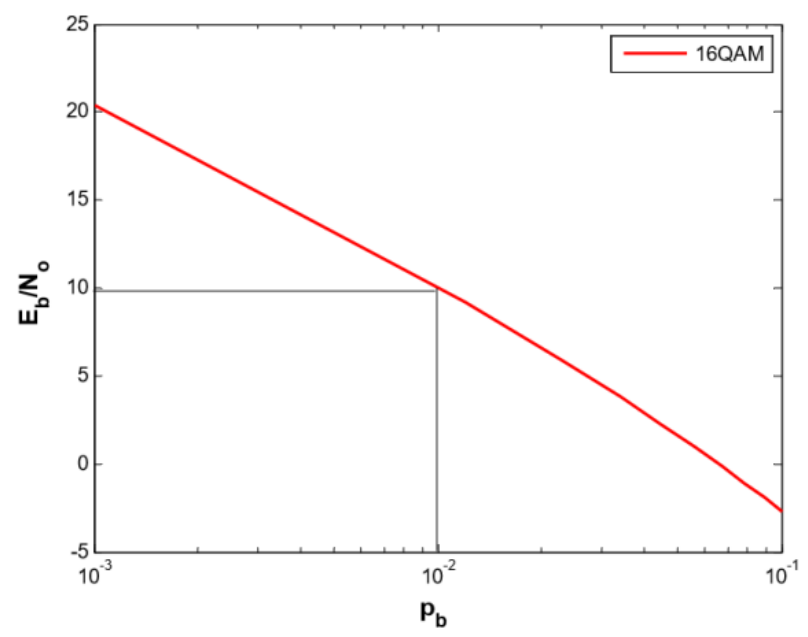

Figure 4: 16-QAM performance for a Rayleigh fading channel. 
The same method was applied to 64-QAM. The result is given by

$\gamma_{b}=10 \log (7)\left(\frac{\left(1-24 / 7 p_{b}\right)^{2}}{48 / 7 p_{b}-576 / 49 p_{b}^{2}}\right)$

which is plotted in Figure 5. As can be seen, $E_{b} / N_{0}=$ $16.4 \mathrm{~dB}$ is required for $p_{b}=10^{-2}$

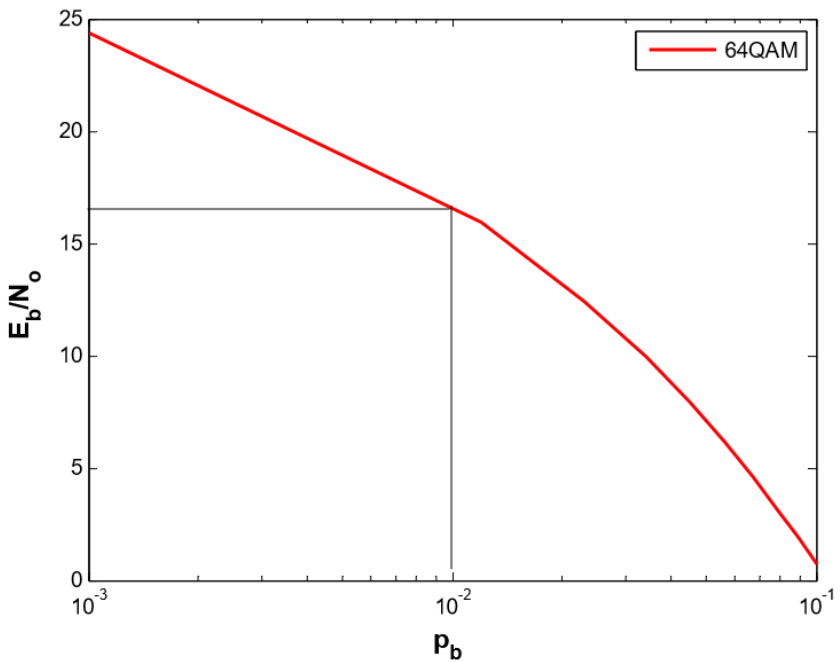

Figure 5: 64-QAM performance for a Rayleigh fading channel.

\section{CCSK}

The probability of channel symbol error for the JTIDS single-pulse architecture is

$p_{s}=\sum_{j=0}^{32} \zeta_{j}\left(\begin{array}{c}32 \\ j\end{array}\right) p_{c}^{j}\left(1-p_{c}\right)^{32-j}$

where the $\zeta_{j} \mathrm{~s}$ are given in Table 2 and is the probability of chip error.

$p_{c}=\left(\frac{1}{2}\right) e^{-5 \gamma_{b} / 32}$

For the $(31,15)$ RS code used in JTIDS, the probability of information bit error is given by [4]

$p_{b} \approx \frac{0.6}{31} \sum_{i-9}^{31} i\left(\begin{array}{c}31 \\ j\end{array}\right) p_{s}^{i}\left(1-p_{s}\right)^{31-i}$

which is plotted in Figure 58. From Figure 58, we see that $p_{s}=0.058$ is required to achieve $p_{b}=10^{-5}$. Note that knowledge of the specific error correction coding used allows us to obtain a JTIDS-specific result.

Figure 6: JTIDS probability of info bit error vs. probability of channel symbol error.
Table I: Conditional probabilities of channel symbol error $\zeta_{j}$ for JTIDS [6].

\begin{tabular}{|l|l|}
\hline $\boldsymbol{J}$ & \\
\hline 0 & 0 \\
\hline 1 & 0 \\
\hline 2 & 0 \\
\hline 3 & 0 \\
\hline 4 & 0 \\
\hline 5 & 0 \\
\hline 6 & 0 \\
\hline 7 & 0.0015 \\
\hline 8 & 0.0221 \\
\hline 9 & 0.1126 \\
\hline 10 & 0.3669 \\
\hline 11 & 0.7093 \\
\hline 12 & 0.9351 \\
\hline$\ldots \ldots$. & $\ldots$ \\
\hline 32 & 1.0 \\
\hline
\end{tabular}

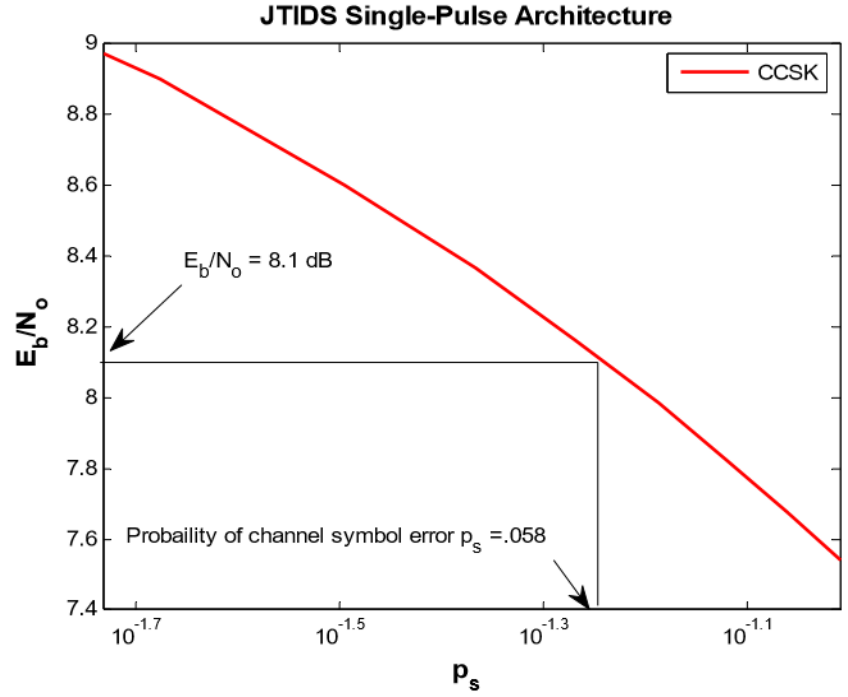

Figure 7: JTIDS performance as a function of probability of channel symbol error.

(B) Results in MATLAB environment

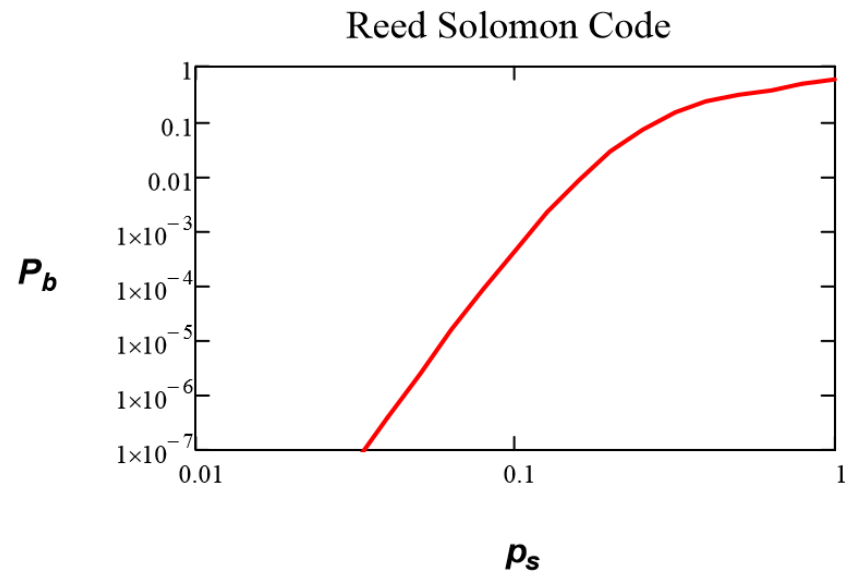

In this section, considering M-QAM modulation technique to transmit the signal and AWGN white Gaussian noise is also introduced in the Rayleigh multipath fading channel in 
digital communication. This section includes plot of random signal, scatter plot of constellation diagram and plot of power of faded signal.

\section{CONSTELLATION DIAGRAM}

In this section, plot shows received faded and noisy signal for various size of constellation.

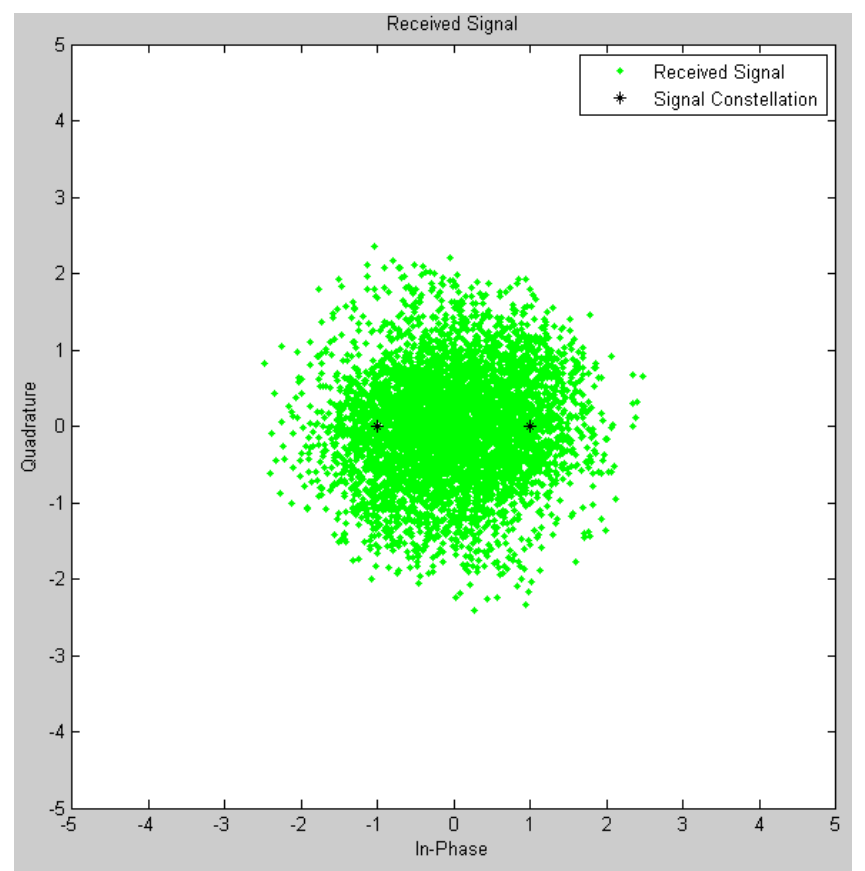

(a)

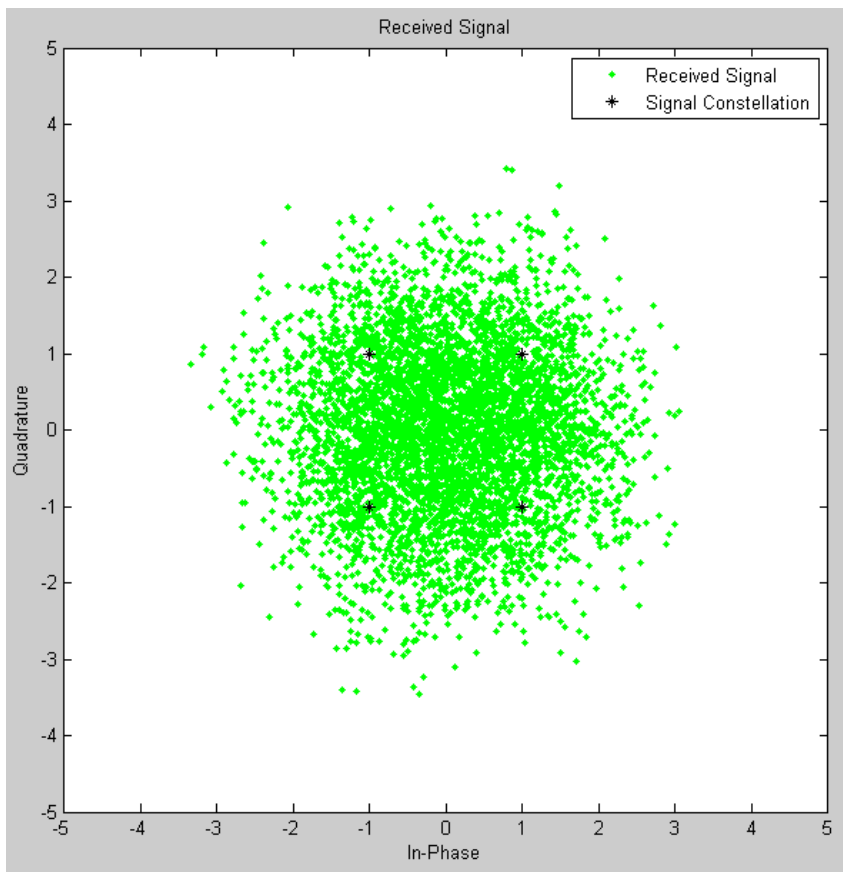

(b)

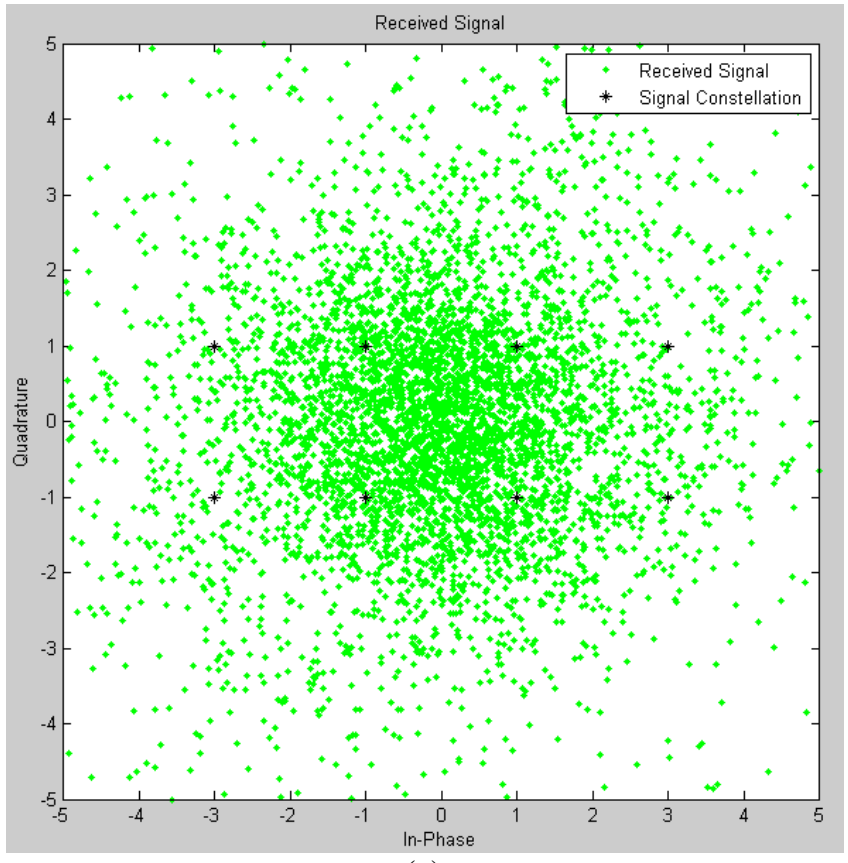

(c)

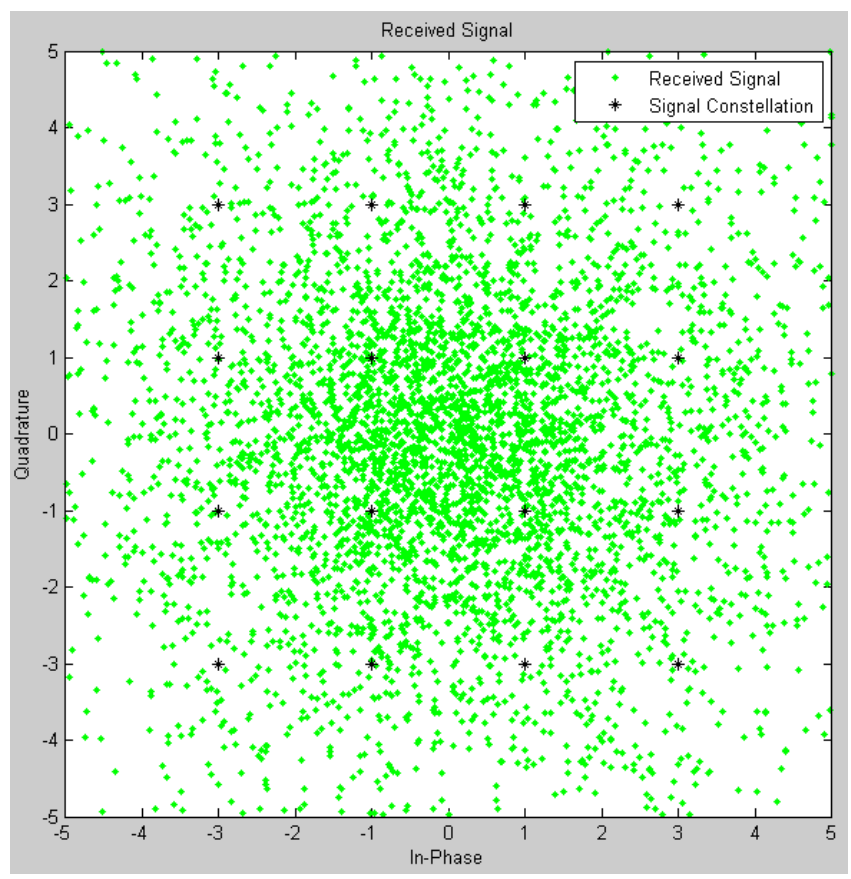

(d)

Figure 8: Constellation diagram after introducing AWGN channel and Rayleigh fading for (a) $m=2$ (b) $m=4$, (c) $m=8$ and (d) $m=16$ 


\section{Power of faded signal}

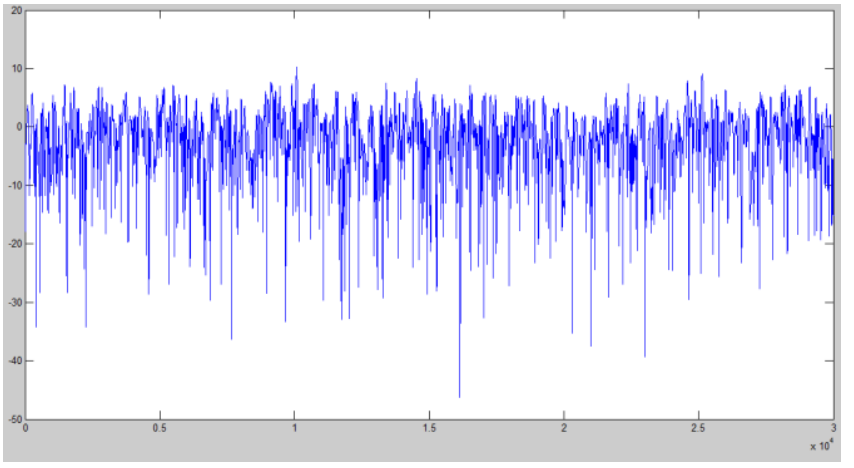

(a) $\quad m=2$

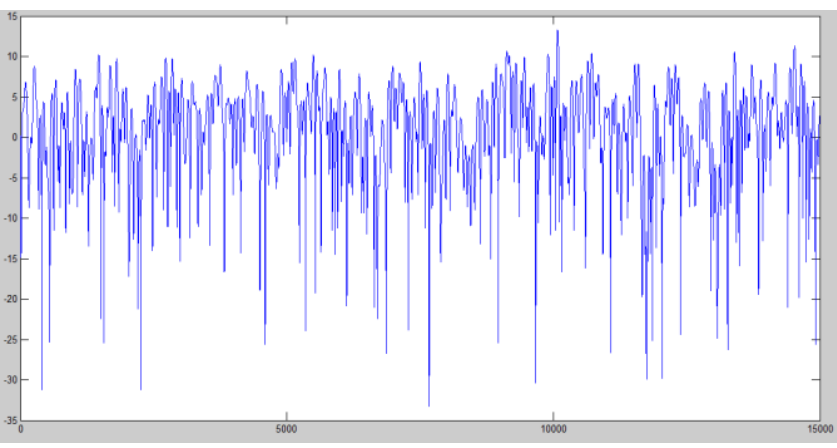

(b) $m=4$

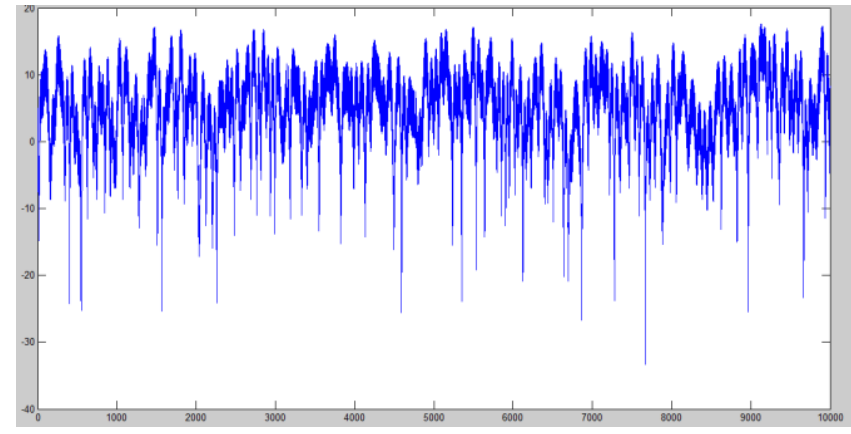

(c) $m=8$

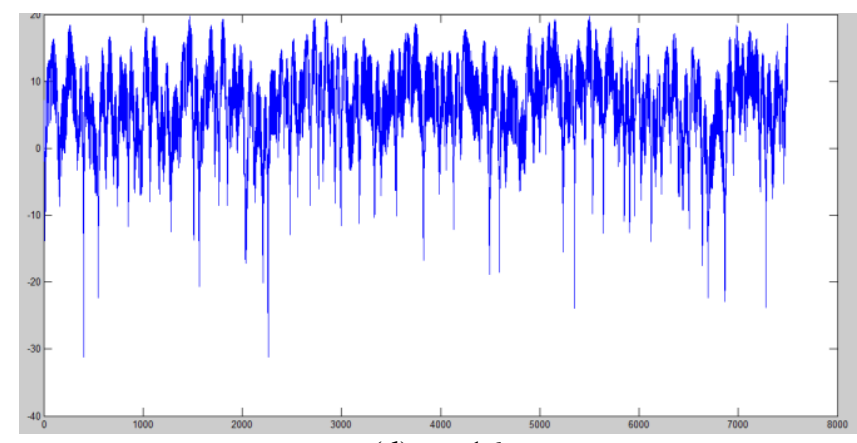

(d) $m=16$

Figure 9: Power of faded signal for (a) $m=2$ (b) $m=4$, (c) $\mathrm{m}=8$ and $(\mathrm{d}) \mathrm{m}=16$

\section{CONCLUSION AND FUTURE WORK}

The results obtained in this paper will ultimately be used in the link budget analysis equation to give users of IMOM a greater sense of situational awareness when interpreting data provided by the software. The modulation techniques evaluated are generic and yet are intended to give a baseline for more specific applications that this method could ultimately provide. Many of the commonly used digital modulation techniques for wireless communications and military applications were evaluated in this paper. In this paper we also simulate constellation and power of faded signal for the one modulation method in MATLAB environment. Similarly, results can be obtained for remaining modulation techniques.

\section{REFERENCES}

[1]. Bernard Sklar, "Rayleigh Fading Channels in Mobile Digital Communication Systems Part I: Characterization", IEEE Communications Magazine, 0163-6804/97 (C) 1997 IEEE.

[2]. J. H. Schiller, "Mobile Communications", Addisonwesley, 2000

[3]. T. S. Rappaport, Wireless Communications, 2nd Edition, Prentice Hall, 2002.

[4]. J. G. Proakis, "Digital Communications", Fourth Edition, 2001.

[5]. Chi-Han Kao, Clark Robertson, and Kyle Lin, Performance Analysis and Simulation of Cyclic CodeShift Keying, to be published in Proc. of IEEE Military Communications Conference, 2008.

[6]. R. Clark Robertson and Nathan E. Beltz, Digital Communications Over Fading Channels, Naval Postgraduate School, Monterey, CA, Nov 2004.

[7]. Clark Robertson, Notes for EC4550 Digital Communications, Naval Postgraduate School, Monterey, CA, 2007. 